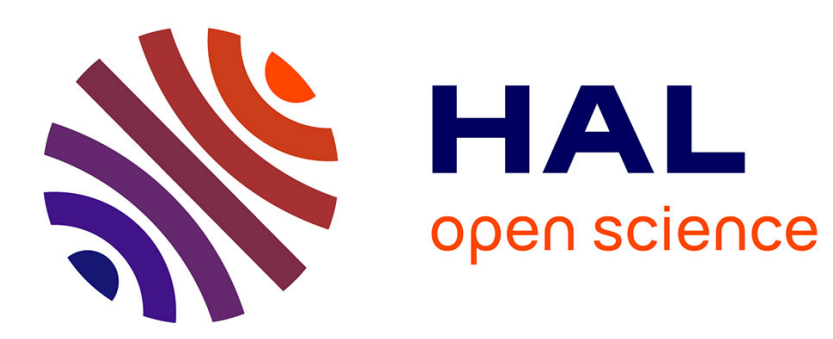

\title{
Support médico-social et éducatif : devenir de familles très carencées confrontées à l'alcool
}

\author{
Annick Camille Dumaret, Marie Constantin-Kuntz
}

\section{To cite this version:}

Annick Camille Dumaret, Marie Constantin-Kuntz. Support médico-social et éducatif: devenir de familles très carencées confrontées à l'alcool. Pratiques Psychologiques, 2005, 11, pp.85-100. halshs00353042

\section{HAL Id: halshs-00353042 \\ https://shs.hal.science/halshs-00353042}

Submitted on 14 Jan 2009

HAL is a multi-disciplinary open access archive for the deposit and dissemination of scientific research documents, whether they are published or not. The documents may come from teaching and research institutions in France or abroad, or from public or private research centers.
L'archive ouverte pluridisciplinaire HAL, est destinée au dépôt et à la diffusion de documents scientifiques de niveau recherche, publiés ou non, émanant des établissements d'enseignement et de recherche français ou étrangers, des laboratoires publics ou privés. 
Paru dans Pratiques Psychologiques, 2005, 11, 85-100.

\title{
Support médico-social et éducatif :
}

\section{devenir de familles très carencées confrontées à l'alcool}

\section{Annick-Camille DUMARET ${ }^{1}$ et Marie CONSTANTIN-KUNTZ ${ }^{2}$}

\author{
${ }^{1}$ Psychologue, ingénieur de recherche à 1'INSERM, CERMES \\ Centre de Recherche Médecine, Sciences, Santé et Société, Site CNRS, 7 rue Guy \\ Môquet, 94801 Villejuif Cedex. \\ ${ }^{2}$ Psychologue, Centre Médico-Psychologique de Courbevoie, \\ 1 Avenue Jeanne, 92600 Asnières
}

Coordonnées de 1'auteur correspondant : dumaret@vjf.cnrs.fr

\section{Résumé français}

Des stratégies de «rencontres» entre familles, professionnels et bénévoles, ayant pour but d'aider les parents à rompre avec l'exclusion sociale et de développer leurs compétences parentales, ont été évaluées. Vingt deux familles très carencées, porteuses de nombreux facteurs de risque dont 1'alcoolisme familial trans-générationnel, ont été interviewées sept ans en moyenne après la fin d'un support médico-social et éducatif. Elles avaient participé avec leurs jeunes enfants à un groupe convivial informel pendant une moyenne de 30 mois. L'article est centré sur la situation actuelle des parents, leurs compétences sociorelationnelles; les compétences verbales des mères ont également été analysées. Trois profils de familles ressortent des analyses, selon leur autonomie et selon qu'elles élèvent ou non leurs enfants. Un tel accompagnement précoce et de longue durée est à mettre en parallèle avec la très forte diminution des violences intra-familiales et la rupture avec le cycle de la maltraitance et des placements ; la 
moitié des familles sont devenues autonomes, neuf mères sur onze ont cessé toute addiction à l'alcool. Néanmoins, le support des professionnels reste indispensable auprès des familles les plus démunies.

\section{Mots clés}

Précarité, familles à risque, alcool, déficience intellectuelle, support social, action médico-sociale

\section{Summary}

This research has evaluated the impact of a group support designed to help parents to face social isolation, to be responsible of their health and to enhance parental competencies. Twenty-two families from high risk backgrounds with a prevalent history of transgenerational family alcoholism were assessed through a psychological and comprehensive approach. Families with their infants or toddlers participated for more than one year to the group support which included professionals and paraprofessionals too. Evaluation took place seven years on average after receiving such a support: all the mothers and eight spouses were interviewed. This paper focuses on the current situation of the parents, their social relationships competencies and their parenthood. The verbal competencies of the mother were also assessed. Three family profiles were drawn from this analysis, based on their level of autonomy and depending on whether they were raising their child or not. The impact of such an early intervention is notable among these families: intra-family violence has largely diminished, severe neglect and abuse has disappeared, nine mothers out of eleven became alcohol abstinent. An increased level of autonomous in half of the families was also observed. However, some situations remain precarious and support from social workers is absolutely necessary for very disabled families from high risk environments.

\section{Key-words}

Precariousness, families at risk, alcohol, déficiencies, early intervention 
Des institutions travaillant en pédopsychiatrie ou en santé communautaire accompagnent des parents confrontés à des carences multiples. Visant à prévenir la maltraitance et les placements, certaines sont axées sur le traitement des troubles de la parentalité et le développement des potentialités de l'enfant. Avant même la loi de janvier 2002 sur la nécessité d'évaluer les pratiques professionnelles, plusieurs d'entre elles ont désiré évaluer leurs actions. Intégrée dans le cadre d'un programme de recherches mené au CERMES, une étude a été mise en place en 1999 au Centre d'Action Médico-Sociale Précoce (CAMSP) de Roubaix. Il s'agissait d'estimer les effets d'une de ses activités, le groupe du mardi composé de professionnels, de bénévoles et de familles. Ce groupe a créé des stratégies de rencontres s'adressant à des familles vulnérabilisées, parfois depuis plusieurs générations, par l'accumulation de difficultés économiques, de problèmes de santé physique et mentale, avec une forte prévalence d'addiction à 1'alcool. L'étude avait pour objectif d'analyser le devenir des parents et de leurs enfants. Cet article se propose d'en présenter certains aspects, centrés sur 1'insertion socio-relationnelle des familles, le fonctionnement cognitif des mères et la parentalité.

\section{Contexte théorique}

De nombreuses études ont montré que pauvreté économique, isolement sociorelationnel, vécus traumatiques, alcoolisme parental et maladie mentale sont associés aux négligences graves et aux maltraitements (Crittenden 1988, Pianta et 
col. 1989, Dinwiddie 1993, Cadzow et col. 1999, Kumpfer et col. 1995). Ces variables, notamment la maladie psychiatrique et la déficience cognitive parentale, sont à mettre en relation avec les problèmes de parentalité et un taux élevé de placement des enfants (Seagull et col. 1986, Tymchuk et Andron 1990, Withman et Accardo 1990, Svedin et col. 1996, Oyserman et col. 2000). Même si elles n'aboutissent pas au placement, d'importantes difficultés éducatives ont été soulignées chez les parents présentant des déficiences cognitives : interactions parents-enfants moins nombreuses, punitions plus importantes que dans les autres familles (Tymchuk 1990, Ethier et col. 1999, Coppin 2004). Ce constat a également été effectué dans les milieux pauvres (Zaouche-Gaudron et col. 1997). Néanmoins, quel que soit le milieu, l'isolement et le manque de support social représentent autant de facteurs de mal être (Llewellyn et col. 1999, Pan Ke Shon 2003) et de facteurs pathogènes dans la relation parent-enfant (Booth et Booth 1993, Boukobza 1999, Guédeney et col. 2000).

Tenant compte de ces constats, des supports sociaux ont été fournis dans le cadre de programmes d'interventions précoces, avec en particulier des groupes de parents. Ils montrent des influences positives sur les compétences parentales et sur la qualité des relations parents-enfant (Seitz et col 1985, Barnard et col., 1988, Black et col., 1994, Fraser et col. 2000). Leur efficacité parmi certains types de populations (déficience cognitive, toxicomanies, problèmes de santé mentale) était plus importante quand des services concrets et cohérents étaient proposés aux familles (halte-garderies, transports...) et quand ils avaient débuté en période prénatale. En particulier, l'étude de Olds et col. $(1995,1999)$ en milieux sociaux 
précaires montre à la fois moins d'hospitalisations en urgence et de placements pour les enfants, moins de stress chez les parents et une meilleure adaptation des mères (moins de recours aux aides sociales, reprise d'activité professionnelle...). Celle de Ernst et col. (1999) auprès de mères addictées à 1'alcool montre aussi l'importance du soutien social pour permettre à ces femmes de renouer avec les services sociaux et les services de soins.

Parmi les facteurs de réussite, les auteurs mentionnent la relation positive entre les intervenants et les parents, relation basée sur la confiance réciproque et le respect des valeurs et de la culture des familles. L'efficacité de ces prises en charge précoces reste toutefois limitée à long terme (Dumaret 2003a). En effet, face à des situations de pauvreté persistante et de cumul de dysfonctionnements transgénérationnels, un accompagnement médico-socio-éducatif de plusieurs mois se révèle insuffisant (Goodman et Johnson 1986, Chaffin et col. 2001). Le travail sur le support social et la parentalité est primordial et doit être poursuivi sur plusieurs années (Molénat et Toubin 1996). Pour consolider l'émergence des compétences des parents et des enfants, ces premières interventions doivent être poursuivies par des stratégies de travail en réseau sur le long terme.

\section{Contexte institutionnel}

Comme tous les CAMSP, le CAMSP de Roubaix est un centre médico-social spécialisé dans la prise en charge précoce (0-6 ans) des enfants. Son équipe est pluridisciplinaire: pédiatre, médecin de réadaptation fonctionnelle, 
neuropsychiatre, psychologue, kinésithérapeute, assistante sociale, psychomotricienne, orthophoniste, éducatrices... Il travaille en partenariat avec d'autres équipes médicales et sociales (Protection Maternelle et Infantile, Aide sociale à l'enfance ASE, pouponnières, crèches, écoles, services de soins psychiatriques pour adultes...) et celles de la Justice. Sa spécificité tient à la population prise en charge : installé depuis 1981 dans un quartier très défavorisé, un grand nombre des familles qu'il reçoit est porteur de vulnérabilités multiples (Potekov et Titran 1996). Aussi, la nécessité de prendre en considération l'environnement social des familles s'est rapidement imposée à l'équipe qui s'est rendue compte que la multiplicité des intervenants et la parcellisation des propositions de prise en charge avaient conduits certains parents à s'exclure des dispositifs d'aide. Avec le temps, l'équipe a ainsi appris à penser la manière de les accueillir.

Sur la base de ces réflexions, un lieu de rencontres entre familles, professionnels et bénévoles a été créé il y a plus de 15 ans. Conçu comme un groupe informel de rencontres sur un mode convivial, il a pour objectif d'être un lieu de connaissance et de reconnaissance de l'autre (Titran, 1993). Sa finalité est d'aider ces familles sans repères à (re)trouver confiance dans les professionnels, d'essayer de restaurer une identité sociale, une image de soi «suffisamment bonne » et de valoriser les compétences parentales par de petits actes ordinaires. Les échanges permettent le partage des compétences entre participants d'un côté et professionnels et bénévoles de 1'autre, qui fera émerger une stratégie d'action pour les familles. Ainsi, grâce au groupe, certaines pourraient prendre en charge 
leur propre santé (consultation en alcoologie, en néonatologie...) et s’impliquer dans l'élaboration d'un projet pour leur enfant (Titran, 2004).

Pour atteindre ces objectifs, des modalités de fonctionnement ont été définies. En premier lieu, un cadre a été mis en place; il constitue une enveloppe structurante et bienveillante qui permettra aux familles de retrouver des repères. Sur le plan pratique, le groupe a lieu un après-midi par semaine, tout au long de l'année. Un système de voiturage est prévu pour les parents ne pouvant utiliser les transports en commun. Les mères et leurs enfants sont au cœur du groupe, mais les pères y sont également conviés ainsi que parfois des grands-mères. Les familles viennent à leur rythme et la fréquentation du groupe s'arrête quand l'enfant a six ans. Le groupe est très tolérant à l'égard des comportements des parents, sauf en cas de violence. Les professionnels et les bénévoles qui accompagnent ces familles sont toujours les mêmes.

Le groupe du mardi se déroule autour d'activités de la vie quotidienne (fabrication de petits objets, couture, préparation de repas...); des sorties et une semaine de vacances l'été sont par ailleurs organisées avec les participants. Les activités manuelles constituent un partage de temps et de savoir, permettent de faire valoir les compétences de chacun et libèrent la parole qui circule entre les participants. Le groupe du mardi peut aussi être un lieu où les parents retrouvent leurs enfants placés par décision judiciaire. Une meilleure compréhension réciproque peut ainsi s'établir et les parents peuvent faire l'expérience de relations sociales satisfaisantes 
L'observation des interactions mère-enfants permet aux professionnels d'identifier les besoins des familles. Une réunion de synthèse entre professionnels et bénévoles a lieu à la fin de chaque groupe et donne l'occasion de faire le point sur l'évolution de l'enfant (cahier individuel pour chaque famille).

\section{Matériel et méthode}

\subsection{Hypothèses}

Comme l'ont montré les travaux cités, il y a lieu de supposer que 1'accompagnement médico-socio-éducatif précoce et de longue durée, proposé par le groupe du mardi, permettra à des parents non seulement à faire face à leur problème d'addiction à l'alcool, de sortir de l'isolement social mais aussi de (re)découvrir leur fonction parentale. On peut penser que la qualité des interrelations qui se sont créées dans le groupe au fil du temps entre parents et intervenants sera à l'origine de la sortie de l'exclusion sociale. Nous supposons aussi que les négligences graves auront diminué.

\subsection{Sélection de la population d'étude}

La méthodologie est celle qui a été utilisée dans le programme de recherche du CERMES (Dumaret 2004a, Dumaret et Titran 2004). Il s'agit d'une étude prospective dans le passé : 24 familles ont été rigoureusement sélectionnées selon les critères suivants : 1) les parents ont participé aux activités du groupe entre 1989 et 1995, pendant une durée d'un an et plus ; 2) l'enfant a été pris en charge tôt (avant 4 ans et demi) ; 3) l'évaluation a été réalisée plus de cinq ans après que 
les familles aient quitté le groupe. Deux familles, uniquement concernées par une pathologie psychiatrique maternelle très lourde sans notion d'abus d'alcool au sein de la famille, ont été exclues. Au final, la recherche porte ainsi sur 22 familles, toutes confrontées à l'alcoolisation parentale ou grandparentale.

\subsection{Recueil des données et analyses}

L'approche de ces familles vivant dans des situations extrêmement précaires a été difficile (changement de nom, détérioration des boîtes à lettres, rendez-vous manqués ou oubliés). Néanmoins, toutes les mères et huit pères ou conjoints ont été rencontrés par un chercheur extérieur à l'institution (A.C. Dumaret). La majorité des interviews ont été effectuées à domicile et les entretiens ont été enregistrés avec leur accord.

Deux évaluations standardisées ont été réalisées : un questionnaire de santé (Nottingham Health Profile, NHP) venant compléter le discours des parents et quatre subtests de la WAIS-III (information, compréhension, vocabulaire et similitudes). Ces subtests verbaux ont été choisis car les travaux scientifiques montrent tous les effets de l'addiction à l'alcool sur les épreuves verbales. L'indice de compréhension verbale ICV (Vocabulaire, Similitudes et Information) est une estimation du QI verbal des mères, car très corrélé avec le QIV ( $r=.94$ ) et également avec le QIG ( $\mathrm{r}=.85$ ) (Manuel de Wechsler, 2000, p338). Toutes les mères ont accepté de se livrer à cette investigation sauf une. Rappelons la signification de ces subtests (Bourgès 1992) L'épreuve «Information » souligne l'influence du milieu socio-culturel et l'attention à la vie sociale. Très dépendant des contextes scolaire et socio-culturel, le subtest « Vocabulaire » renvoie en plus, 
sur le plan affectif, à l'expression verbale en tant que composante du langage relationnel. L'épreuve «Compréhension » est indépendante des acquis scolaires qui s'appuie sur l'intelligence intuitive, la capacité à réagir de manière réflexe. « Similitudes »: indépendant des influences socio-culturelles, ce subtest, moins soumis à l'aisance verbale, est l'un des plus représentatifs du QI et met en jeu trois capacités : la conceptualisation, la généralisation (facteur $G$ ) et la pensée catégorielle.

L'évaluation qualitative a été effectuée à partir d'entretiens semi-directifs au cours desquels ont été abordés l'histoire familiale, la vie socio-professionnelle et relationnelle, les souvenirs du groupe du mardi (un article précédent a été consacré aux représentations des parents quant au soutien reçu (Dumaret 2003b). La parentalité a été étudiée en référence aux travaux de D. Houzel (1999). Une analyse de contenu des entretiens a permis de synthétiser les données recueillies et de construire plusieurs variables socio-démographiques: les ressources de la famille (salaire, prestations sociales, revenu minimum d'insertion RMI, allocation adulte handicapé $\mathrm{AAH}$ ), le type de famille (mono, biparentale, avec le père de l'enfant ou avec un concubin), le placement ou non de l'enfant. Dans le domaine socio-relationnel, on a tenu compte pour les parents, en plus de 1'existence de loisirs, des liens avec : 1) leurs parents, leurs frères et sœurs, 2) la famille élargie, 3) les amis, 4) le voisinage, 5) les professionnels (tutelle, ASE...). Les relations familiales ont été codées d'inexistantes à aidantes. L'ensemble des données ainsi recueillies a été confronté, aux données de l'institution (dossiers socio-médicaux 
et cahiers du mardi) et aux informations obtenues lors de rencontres avec les référents sociaux (ASE, organismes de tutelle)

Les analyses de données ont été réalisées à partir de tests classiques $\left(\chi^{2}\right.$, analyses de variance, tests non paramétriques, coefficient de corrélation $\mathrm{R}$ de Spearman). La régression linéaire multiple a été utilisée en ajustement sur les facteurs de confusion.

\section{Situation des familles à l'admission au groupe et lors de}

\section{l'accompagnement}

A l'exception de deux mères, aucune ne possédait de diplôme, plusieurs avaient suivi des classes de transition ou un enseignement spécialisé. Aucune mère n'avait d'activité professionnelle. Rares étaient les mères ayant pu bénéficier de figures et/ou de modèles parentaux stables : au moins 18 mères $(82 \%)$ avaient vécu des maltraitances importantes dans l'enfance et l'adolescence ayant nécessité des placements à 1'ASE. Quatorze mères sont porteuses de séquelles d'alcoolisation fœtale (tableau 1). La dépendance à 1'alcool (11 mères) est associée à des situations d'errance, des maltraitances sexuelles (5 mères en ont parlé) et une pathologie psychiatrique : dans la plupart des cas, cette dernière a pu être précisée en fonction des critères diagnostiques du DSM IV sur la base des résumés de rapports d'hospitalisation et/ou de suivi psychiatrique extraits des dossiers institutionnels. Dans 17 familles, la violence entre conjoints et/ou envers les enfants avait été soulignée. 
Tableau 1

Les parents sont venus au groupe avec leur(s) enfant(s) en moyenne 30 mois. Ce sont les mères présentant le plus de problèmes de santé mentale qui ont participé proportionnellement le plus longtemps au groupe car elles ont continué à venir seules, sans enfant, encore plusieurs mois au groupe. Trois mères très déficientes intellectuellement sont venues irrégulièrement et deux mères ont été exclues.

\section{Profil actuel des familles : vie socio-professionnelle et relationnelle}

Le devenir des familles a été réévalué en moyenne sept ans après la sortie du groupe. L'âge moyen des mères est de 40 ans. Trois profils de familles ont été dégagés d'un classement qui tenait compte de plusieurs variables : 1) la situation familiale : autorité parentale, lieu de vie des enfants, 2) les types de revenus, 3) l'état de santé général. Le premier groupe se compose de familles qualifiées d'autonomes et dont les situations parentales se sont nettement améliorées (Aut), le second groupe se compose de familles stabilisées (Stab) aux situations légèrement améliorées. Enfin, le dernier groupe concerne des situations inchangées (Inc). Un tel classement s'est justifié a posteriori. En effet, les résultats d'une analyse de régression multiple, avec comme variable dépendante, les trois profils de famille, et comme variables indépendantes : les ressources principales de la famille, le type de famille (monoparentale, avec le père ou un concubin), le placement actuel d'enfants à l'ASE et les séquelles d'alcoolisation chez la mère, 
indique que chacune des trois premières variables indépendantes a un effet prédictif statistiquement significatif sur ce classement. Les séquelles d'alcoolisation chez les mères n'empêchent pas une évolution vers l'autonomie.

Sur le plan de la santé perçue (NHP), des différences apparaissent entre les trois groupes de famille en ce qui concerne la sphère émotionnelle et la solitude. Les mères Stab, qui présentent plus de dysfonctionnements psychiques, ont beaucoup plus de troubles émotionnels que les autres mères et se sentent plus isolées (plusieurs prennent des neuroleptiques et des antidépresseurs et sont suivies régulièrement). Par ailleurs, le questionnaire de santé perçue montre que ce sont les mères Aut qui se sentent les moins isolées.

C'est pendant la participation au groupe que des mères ont entrepris une (ou plusieurs) cure de désintoxication: "J'avais la sécurité de ne plus être seule, d'avoir un peu de bien-être comme si on trouvait un peu de la famille... Y avait pire que moi. J'ai décidé d'arrêter en voyant quatre mères alcooliques, leurs enfants étaient placés. " La peur du placement ou de ne plus récupérer l'enfant placé est la première raison mise en avant. D'autres, confrontées à des mères très addictées ou qui s'en étaient sorties, se sont senties épaulées et encouragées : « $A$ l'époque je savais que je buvais, sur les photos, j'avais le nez rouge, ils ont pas dî le voir. Y avait une maman, elle venait, elle était éméchée, je me suis dit, pour une femme, c'est dégoûtant de boire, c'est pas beau une femme qui boit. Je dois être pareil, il vaut mieux arrêter.» La disparition de l'alcoolisme maternel est le facteur déclenchant l'amélioration de la vie quotidienne; les couples violents se sont séparés, des mères ont trouvé un nouveau concubin (tableau 1). 
Tableau 2

Ainsi que 1'indique le tableau 2, l'analyse des relations familiales et amicales montre que si les parents entretiennent des liens plus ou moins réguliers avec leur parentèle, c'est l'existence de relations amicales pour les parents, le fait d'avoir des loisirs, de savoir demander de l'aide ainsi que d'avoir peu d'intervenants professionnels au sein de la famille qui distingue les trois profils familiaux, les parents autonomes étant les plus ouverts au monde extérieur.

\subsection{Les familles autonomes $(n=12)$}

Toutes les mères sauf une sont devenues abstinentes. La moitié des familles ont au moins un salaire comme ressource principale, quatre mères qui n'avaient jamais travaillé ont une activité professionnelle. Une autre mère a obtenu un diplôme d'aide-soignante. Les conjoints sont très aidants, l'isolement social a fortement régressé (seules trois familles vivent encore assez isolément), le réseau relationnel est plutôt diversifié.

\subsection{Les familles stabilisées $(n=6)$}

L'entourage familial et amical est plus restreint, les loisirs sont plus liés aux enfants et moins variés que dans le groupe précédent. Les deux tiers des familles se sentent isolées, d'autant plus que leurs enfants sont pris en charge par 1'ASE. Les ressources principales sont en majorité l'AAH.

\subsection{Les situations familiales inchangées $(n=4)$}

les familles vivent autant du RMI que de l'AAH, l'endettement est toujours présent. Parmi les trois mères alcooliques, deux disent être devenues abstinentes. 
Trois des conjoints sont alcooliques. Sans entourage familial ou amical, le seul support existant est celui des professionnels, très présents dans les familles.

\section{L'estimation du fonctionnement cognitif des mères}

La note moyenne obtenue par 21 mères à l'indice de compréhension verbale, qui fournit une bonne approximation du QIV, est de $68 \pm 11,3$. Les moyennes des notes standards aux quatre épreuves passées (Information I, Vocabulaire V, Similitudes S, Compréhension C) sont présentées dans le tableau 3. Se situant toutes en-dessous de la moyenne, elles manifestent en premier lieu les faibles compétences des mères sur le plan verbal.

Tableau 3

L'analyse du contenu des réponses fait apparaître d'autres difficultés au niveau du fonctionnement cognitif, au premier rang desquelles d'importantes difficultés de représentation mentale, avec un recours à la pensée concrète, corollaire à la défaillance des processus d'abstraction et de symbolisation ( $I, V, S)$. Le fonctionnement se situe dans la pensée opératoire.

Par ailleurs, l'échec à l'épreuve Compréhension témoigne des défaillances de l'éducation reçue ou de son intégration et manifeste les difficultés d'adaptation à la vie quotidienne. De plus, la difficulté à évoluer dans son propre environnement ne favorise pas l'intérêt pour d'autres cultures et leur compréhension, ce qui s'exprime au long des quatre épreuves à travers des réponses au référentiel culturel ego-centré : réponses à 1'item $5(\mathrm{C})$ : "savoir ce qui s'est passé dans 
notre région ", " pour que les enfants sachent comment c'était dans notre temps à nous ». Les définitions données par les mères tournent ainsi autour du vécu. Les résultats mettent en évidence la grande difficulté des mères à porter une attention à une vie sociale dont les frontières dépassent celles de leur activité quotidienne. Même lorsque les questions font appel à des capacités d'abstraction et de décentration, comme par exemple les items à contenus culturels $(I, V, C)$, elles les ramènent par leurs réponses au concret de leur espace-temps, elles restent ancrées dans le quotidien et le familier, dans une approche qui apparaît ego-centrée.

Toujours en référence à leur propre expérience, l'intérêt porté au corps et à la maladie, la saleté et la propreté, le vécu de la violence et les références aux transgressions de la loi sont très présents. Les réponses présentant un tel contenu, à forte valeur projective, manifestent par ailleurs souvent une approche immature des situations. Ainsi, si la moitié des mères semblent avoir intégré le discours sanitaire et diététique, vraisemblablement lié au soutien socio-éducatif, les autres témoignent d'une pensée enfantine basée sur la sensorialité : réponse à l'item 4 (C) : « c'est pas bon ». En ce qui concerne les réponses faisant référence à la loi, elles signalent un positionnement enfantin face celle-ci, sur le modèle de l'élève face à son maître. D’autres réponses renvoient au vécu et à la représentation de 1'école chez ces mères : difficulté d'investissement de la scolarité et école non perçue comme garante de l'égalité des chances et donc comme facteur d'intégration sociale. Dans ce contexte, il n'est pas surprenant que l'école puisse être assimilée à l'institution judiciaire, elle-même souvent vécue comme persécutrice : " on se fait toujours avoir avec eux 》. 
Pour pallier les défaillances d'accès à la symbolisation et à l'abstraction, certaines stratégies semblent préférentiellement utilisées. Par exemple, les réponses données sont parfois partielles sans être forcément inexactes, avec des sous-entendus non verbalisés. Le geste est parfois utilisé, le langage ne pouvant servir de contenant à la pensée. L'association par sonorités se retrouve également: lorsqu'elles ne comprennent pas un mot, les mères interrogées donnent une réponse se rapportant à un mot phonétiquement proche : réponse à 1'item 11 (I) : «l'eau dans le courant est nue ».

Ainsi, de façon globale, le mode d'approche apparaît caractérisé par le recours à un référentiel limité à l'environnement proche. Dans ce registre, certaines thématiques sont récurrentes: la référence au corps, en particulier au corps malade, la violence et l'agressivité subie ou donnée, ainsi que l'évocation de l'autorité judiciaire et des infractions qu'elle réprime. Nombre de ces réponses présentent une forte dimension projective qui se confirme lorsqu'on les croise avec certains éléments biographiques des mères. Ainsi, la grande majorité des réponses $(93 \%)$ faisant référence à la violence est le fait de mères 1'ayant ellesmêmes subie dans l'enfance (agressions sexuelles, maltraitements, placements) et/ou de celles dont la famille est suivie en AEMO (agressions sexuelles envers enfant, etc...). Les mêmes antécédents de violence se retrouvent pour $91 \%$ des réponses faisant référence à la justice. De même, les réponses faisant appel à des connaissances culturelles sont fréquemment échouées, car ramenées à un espace culturel ego centré. Ce processus manifeste une défaillance de la transmission trans-générationnelle qui explique un fonctionnement cantonné dans la pensée 
concrète et opératoire. L'apprentissage scolaire n'est pas venu pallier cette défaillance primordiale. En conséquence, des notions telles que l'espace tridimensionnel, 1'historicité, la chronologie... apparaissent difficiles à appréhender voire absentes pour ces mères. Pour les mêmes raisons, les normes sociales et les règles de vie en communauté se révèlent lacunaires.

\section{Parentalité et relations parents-enfants}

Actuellement, les parents élèvent 62 enfants (d'âge scolaire) parmi les 98 qui constituent leur descendance. Pour ces enfants mineurs, 1'autorité parentale est partagée entre les parents dans six des 22 situations, elle appartient à la mère dans 13 cas. Dans une famille, c'est le père seul qui a l'autorité parentale et dans une autre famille, l'autorité parentale appartient au père pour l'un des deux enfants et à la mère pour 1'autre. Dans le tiers des familles, les enfants étant placés $(n=17)$, les droits de l'autorité parentale sont partagés avec 1'ASE.

\subsection{L'analyse des entretiens avec les parents : généralités}

Cette analyse met en évidence une grande pauvreté du discours. Les valeurs sur lesquelles ces parents fondent leur système éducatif apparaissent conformistes. Elles s'inscrivent dans une recherche de désirabilité sociale (politesse, propreté, respect des anciens, etc.) qui cependant échoue dans la mesure où ce conformisme affiché renvoie à une conception obsolète quasi-hygiéniste de l'éducation. Ceci révèle la difficulté de ces mères à intégrer les normes culturelles contemporaines 
de la société dans laquelle elles vivent, difficulté que les résultats aux épreuves de la WAIS, en particulier ceux de l'épreuve Information, permettent de situer dans un registre cognitif. Les aspects relationnels ou ceux renvoyant à l'intériorité sont peu présents. Cette tendance est majorée chez les mères intellectuellement les plus démunies, pour lesquelles l'éducation est quasi-exclusivement envisagée dans ses aspects pratiques : «un rôle d'un parent c'est qu'il doit gérer les sous pour pas dépenser à tort et à travers », "S'occuper des enfants, faire la cuisine, faire leurs soins, leur toilette, les mettre coucher, faire leur repas et tout ça. "

La perception de l'enfant comme un sujet individualisé, ayant des besoins et des désirs personnels, est peu présente dans le discours des parents. Leur approche apparaît ego-centrée, davantage guidée par des éléments projectifs en référence à leur histoire personnelle, que par l'écoute de ce que l'enfant pourrait exprimer: « J'ai du mal à rouspéter ou à donner une punition. J'ai été élevée bizarrement, j'ai été élevée à la dure, j'étais battue. " Au final, l'enfant est souvent présenté comme un support voire un prolongement narcissique, ayant pour mission de donner un sens à la vie de ses parents, de rompre leur solitude et leur ennui, de leur donner une utilité et une place dans le monde. Ceci se manifeste par des relations majoritairement situées dans un registre fusionnel, avec une incapacité massive à poser des limites intergénérationnelles : « Même quand ils (les enfants) font des bêtises, c'est nous qui a les problèmes "; "C'est mon bonheur d'avoir mes enfants, j'ai toujours voulu rester avec mes enfants seule. Parce que c'est mes yeux, mes enfants, c'est ma vie, c'est ma chair... c'est tout ». Cette confusion 
se traduit par une parentification importante des enfants et peut aller jusqu'à un climat familial incestuel.

Dans ce contexte d'investissement à dominante narcissique de l'enfant, l'avenir est souvent peu évoqué. La séparation future apparaît difficile à envisager : « Faut toujours être derrière eux, quand ils grandissent et quand ils se marient... Ils ont toujours besoin de leurs parents : quand ils ont des problèmes, pour remplir un papier, pour garder les petits-enfants ».

\subsection{Les profils familiaux, ressemblances et oppositions}

Dans les familles Aut, les parents élèvent tous leurs enfants mineurs ; le parent qui n'a pas la garde voit ses enfants assez régulièrement pour au moins la moitié des familles. Dans une seule situation, des conflits perdurent entre le père, qui vit seul et a la charge de ses enfants, et la mère remariée. Ces parents, qui présentent le moins de retard mental (tableau 1), sont ceux qui apparaissent le mieux percevoir les besoins de leurs enfants. Dans leur discours, ils se distinguent des autres parents en mettant en avant dans le rôle parental l'importance de la communication et des relations affectives, avant l'éducation, la surveillance... Ils parlent de leur plaisir à être parents (activités ensemble, sorties, câlins), évoquent les manifestations d'affections de leurs enfants, sont attentifs à leur bien-être. Ainsi, à l'exception d'une mère refusant tout suivi, orthophonique ou autre, pour ses enfants, tous ces parents ont accepté que leurs enfants rencontrent des psychiatres ou des psychologues : "Mon fils, il avait un mal être... il a pété les plombs, mais je lui ai dit qu'il aurait pu en parler directement, les discussions, ça fait beaucoup de bien. Je hi ai dit : 'si tu as envie d'aller voir un psychologue, on 
va ensemble. Après je te fais voir la route et tu y vas seul'. Ben, quand il a fait sa tentative, il a été entendu par un pédopsychiatre, et il a su dire... Et nous, en tant que maman, il me lance des appels que sans doute je n'entends ou je ne vois pas. Donc, maintenant, on est à trois avec mon fils et le pédopsy ».

Si l'aspect protecteur du rôle parental est nommé, ces familles s'efforcent de le réaliser : elles cherchent à protéger leurs enfants (par rapport au père violent, aux quartiers peu sûrs et dégradés, au racket à l'école), quatre d'entre elles ont demandé une aide éducative pour leurs adolescents. Les parents se préoccupent de la scolarité ; même s'ils ne peuvent aider directement aux devoirs, ils vont voir les enseignants.

Ces parents sont les seuls à s'interroger sur leurs méthodes éducatives et à avoir une certaine capacité d'autocritique, ceci n'est d'ailleurs évoqué que par la moitié d'entre eux. Par exemple, dans une famille où les deux parents ont été placés dans leur enfance, un père se demande : " Est-ce qu'on a bien fait ? Parce que je dis que je les ai éduquées au mieux qu'on peut, dans le respect et l'amour, mais c'est pas évident. On essaie de les conseiller, mais est-ce que ça sert, c'est des conseils de vieux... Moi, maintenant, je dis, en étant enfant, si c'était à refaire, j'aurais pas fait ça. Pourquoi j'ai pas été plus vers mon père pour lui parler? Je me pose encore la question et j'ai pas de réponse parce que j'ai plus mes parents. " Voici également les paroles d'une mère dont les enfants sont élevés par l'ex-mari : "J'ai toujours dit, il faudrait pas que mes enfants passent par où je suis passée et qu'ils iront jamais à la Grande Maison [1a DASS]. Et bien, mes deux grands ont été placés, j'en ai mal, j'en souffre. C'est $80 \%$ la faute 
aux parents. Je suis pas sûre d'avoir été une bonne mère. J'aurais été une bonne mère, j'aurais été plus à l'écoute avec mes enfants. Ils avaient peut-être un problème psychologique, j'ai pas compris leur détresse. ”

Parmi les familles Stab, deux élèvent leurs enfants, les autres ont toutes leurs enfants placés à l'ASE. Les relations mère-enfants sont régulières (week-end ou une fois par semaine chez eux, vacances). Les enfants placés voient leur père plus rarement que dans les familles monoparentales du groupe précédent (décès, éloignement après abus sexuels, incarcération). En ce qui concerne la protection des enfants, la façon d'agir n'est pas en concordance avec leur discours, à la différence du groupe précédent.

Dans ces deux premiers groupes de familles, parmi les difficultés éducatives mentionnées, on note chez les parents l'incapacité à poser des limites et à dire "non" à leurs enfants, ne voulant peut-être pas reproduire la violence parentale connue dans leur enfance, à moins qu'ils ne craignent une résurgence de leur propre violence qu'ils essaient de contenir. Certains s'efforcent de se maîtriser, signalant qu'ils préfèrent fumer ou crier plutôt que frapper: "Oui, je l'ai cogné une fois comme si c'était un garçon. De ce jour là, ça m'a marqué, j'ai dit: 'je crie, je ne lève plus la main'. " On a observé la disparition de la maltraitance et des négligences graves, bien qu'il subsiste encore des violences verbales de la part de certains parents, notamment les mères ayant toujours des troubles psychiques. Si le risque " Alcool " a pu être contenu, il n'en est pas de même de la maltraitance sexuelle: les mères n'ont pu anticiper les conduites de certains familiers, de certains pères. Toutefois, les abus sexuels ont pu être dénoncés à la police par les 
mères elles-mêmes, ce qui aurait été inimaginable cinq ou dix ans plus tôt. Les nouvelles mesures d'AEMO qui ont été mises en place suite à ces affaires l'ont été pour des durée assez limitées, les mères tolèrent aujourd'hui la venue des travailleurs sociaux à domicile. Par ailleurs, elles ont accepté les soins psychiques plus rapidement pour leurs jeunes enfants que pour les aînés ayant vécu des traumatismes identiques: "Au tribunal, j'ai demandé de mettre une aide pour justement euh, parce que je sais très bien que, quand on subit des attouchements comme ça, que les enfants vont pas bien...C'était pour un peu ouvrir, même si ils en parlaient pas à moi, au moins qu'ils puissent en parler à quelqu'un d'autre, et puis ça leur faisait du bien. "

Nous avons constaté que les maltraitances sexuelles chez les enfants des familles Aut n'ont jamais été perpétrées par les pères biologiques mais par des personnes de l'entourage proche: oncle, frère aîné, copain du père ou couple d'amis de la mère, éducateur. Ceci peut être mis en relation non seulement avec le fait que ces familles s'en sortent mieux (maintien du couple, relations familiales...) mais aussi parce que le climat familial apparaît moins incestuel. Dans les familles Stab et Inc, l'agresseur est le plus souvent le père, sur ses propres enfants ou d'autres enfants de sa femme, parfois un frère aîné.

Dans le discours des familles Inc qui n'ont pas évolué positivement, les références à l'éducation sont uniquement dans le concret. Aucun des parents ne perçoit les besoins réels de ses enfants, aucun n'arrive à poser de limites sur le plan éducatif. Le climat familial est souvent incestuel. Les parents sont dans la délégation de leur autorité parentale à l'ASE ou dans la toute puissance : trois 
mères, intellectuellement très démunies, sont sous tutelle ou curatelle, une mère borderline vit et règne au milieu de ses enfants. Les pères sont absents (décès, incarcération, maladie mentale).

\section{Synthèse et discussion}

Les familles dont nous avons étudié le devenir ont bénéficié d'un accompagnement médico-socio-éducatif pendant plus d'un an par un groupe informel composé de parents avec leur(s) enfant(s) en bas âge, de bénévoles et de professionnels du CAMSP de Roubaix et d'institutions extérieures. Elles présentaient des carences graves et de nombreux facteurs de risque souvent intergénérationnels : plus de quatre mères sur cinq avaient été maltraitées dans l'enfance, et si l'on tient compte des violences intra-familiales, la proportion s'élève à 9 mères sur dix. Soulignons que l'évaluation ne présente pas de biais dus à la perte de sujets : toutes les mères $(\mathrm{n}=22)$ ont été rencontrées ainsi que huit conjoints (père ou concubin). La confiance des mères envers le pédiatre et son équipe, s'est élargie au chercheur qui les a rencontrées et avec qui elles ont pu évoquer des souvenirs pénibles de leur histoire familiale et aborder leur situation actuelle, la question des soins et leur rôle parental.

Nous avons noté les difficultés importantes de verbalisation, de représentation et de conceptualisation. Les faibles compétences verbales des mères testées témoigne non seulement du milieu de vie très carencé et défavorisé, mais aussi des séquelles de l'alcoolisation à la génération précédente. Le fonctionnement de 
leur pensée reste très ego-centré, souvent projectif et très figé dans le concret. Les défaillances dans leur éducation se répercutent dans leur système éducatif, éloigné des conceptions actuelles. L'investissement parental est souvent à dominante narcissique et les parents ont du mal à envisager l'avenir et la séparation avec les enfants qui grandissent. Nombre de parents assument leur parentalité totalement, ou partiellement en cas de placement où il faut remarquer que les liens sont maintenus aussi avec les parents ne détenant pas 1'autorité parentale. Les troisquarts des violences intra-familiales ont cessé ; s'il reste encore des violences verbales envers les enfants, la maltraitance grave a disparu, aucun enfant n'a été placé depuis la sortie du groupe, les nouveaux conjoints sont aidants. Ces résultats vont dans le même sens que ceux d'une étude menée auprès de familles très carencées où les soins mères-bébés, soins psychiatriques et psychologiques sont différenciés du soins social (Dumaret et Picchi 2004), l'importance des soins précoces concerne aussi les situations de placement (Dumaret 2004b). Malgré l'absence de groupe « contrôle », on retrouve ici certaines données de la littérature sur les interventions précoces en milieu défavorisé ou déficient sur le plan cognitif : la diminution de la maltraitance, la présence d'un conjoint aidant et du support social dans 1'accès à 1'autonomie et la redécouverte d'une activité professionnelle et la capacité à demander de 1'aide (Erickson et col. 1992, Olds et col. 1995, Egeland et col. 1996).

Cette amélioration observée pour la majorité des familles qui présentaient toutes d'importants facteurs de risque, apparaît liée à l'arrêt de l'addiction à 1'alcool pour les mères qui ont mentionné que l'importance de l'aide du groupe à 
ce sujet : neuf sur onze sont devenues abstinentes. Ceci rejoint les résultats d'une étude a montré que des mères ayant donné naissance à un enfant porteur de séquelles d'alcoolisation fœtale étaient très nombreuses à avoir été maltraitées et présentaient de nombreux problèmes de santé mentale; comparativement aux autres mères, celles ayant cessé tout addiction avaient un meilleur niveau intellectuel, bénéficiaient de plus de support social et amical et d'un conjoint aidant (Astley et col. 2000).

Il ne s'agit pas d'une étude randomisée, cependant on peut mettre en parallèle les évolutions constatées dans les trois types de familles et le support social du groupe du mardi. En effet, le redéploiement des compétences parentales, l'ouverture à la vie sociale est plus nette parmi les familles devenues autonomes, pour lesquelles la fréquentation du groupe avait été régulière. A l'inverse, dans les situations familiales inchangées où les facteurs de risque perdurent, il y a eu rupture ou irrégularité dans l'accompagnement. Cette proportion d'échecs de prise en charge - environ une famille sur cinq - rejoint ce que nous avions trouvé dans le cas des placements familiaux (Dumaret et Coppel-Batsch 1996). Elle semble manifester ici les limites d'une telle action médico-sociale non relayée par d'autres interventions.

\section{Conclusion}

Depuis plusieurs années, grâce à la pédopsychiatrie et la psychiatrie périnatale, l'importance de l'instauration d'un lien social de confiance est reconnue afin de 
permettre le lien thérapeutique. L'alliance créée au CAMSP de Roubaix entre parents, professionnels et bénévoles pendant de nombreux mois a permis à ces familles de sortir de 1'exclusion et pour nombre d'entre elles de développer leurs compétences parentales. Toutefois, eu égard au réseau socio-relationnel ténu et à la pauvreté encore présente pour nombre de ces familles, certains équilibres s'avèrent toujours précaires. L'activité du groupe du mardi, dans un cadre de santé communautaire, correspond à l'une des orientations de la politique familiale annoncée en 1998, celle concernant la création et le renforcement de réseaux d'appui, d'écoute et d'accueil de parents, visant à les accompagner dans leur rôle éducatif. Ces premières prises en charge doivent trouver un prolongement pour consolider l'émergence des acquis dans ces familles touchées par de nombreux problèmes trans-générationnels.

\section{Remerciements}

Les auteurs remercient le Docteur M. Titran, directeur du CAMSP de Roubaix, pour son aide dans l'élaboration de ce travail et pour leur avoir fait part de la richesse de son expérience auprès des familles du groupe du mardi. Ils remercient également Mme B. Dumont pour la part active qu'elle avait pris dans la recherche des familles. Mme Dumont avait participé à la création du groupe du mardi ainsi qu'à son déroulement.

L'étude a été subventionnée par les Conseils Généraux des départements du Nord et du Pas de Calais, par le Conseil Régional du Nord-Pas-de-Calais ainsi que par la Fondation de France.

\section{Références}

Astley, S.J., Bailey, D., Talbot, C., Clarren, S.K., 2000. Fetal alcohol syndrome (FAS) primary prevention through FAS diagnosis: II A comprehensive profile of birth 
mothers of children with FAS. Alcoholism and Alcoholism 35 (5), 509-519.

Barnard, K.E., Magyary, G.S., Booth, C.L., Mitchell, S.K., Spieker, S.J., 1988. Prevention of parenting altercations for woman with low social support. Psychiatry $51,248-253$.

Black, M.M., Nair, P., Kight, C., Wachtel, R., Roby, P., Schuler, M., 1994. Parenting and early development among children of drug-abusing women: effects of home intervention. Pediatrics 94, 440-448.

Booth, T., Booth, W., 1993. Parenting with learning difficulties: Lessons for practitioners. British Journal of Social Work 23, 459-480.

Bourgès, S., 1992. Approche génétique et psychanalytique de l'enfant. Delachaux et Niestlé, Paris.

Boukobza, C., 1999. Etre mère dans des situations de grande pauvreté. In : Poilpot, M.P., Boukobza, C., Causer, J.Y. (Ed.), Etre parents en situation de grande précarité. Erès, Ramonville Saint Agne, pp. 47-61.

Cadzow, S.P., Armstrong, K.L., 1999. Stressed parents with infants: reassessing physical abuse risk factors. Child Abuse and Neglect 23, 845-853.

Chaffin, M., Bonner, B.L., Hill, R.F., 2001. Family preservation and family support programs : child maltreatment outcomes across client risk levels and program types. Child Abuse and Neglect 25, 1269-1289.

Coppin, B., 2004. Etre parent avec une déficience intellectuelle. Pratiques Psychologiques 10 (1), 25-38.

Crittenden, P., 1988. Family and dyadic patterns of functioning in maltreating families. In : Brown, K., Davies, C., Stratton, P. (Ed), Early prediction and prevention of child abuse. John Wiley \& Sons, New York, pp.161-184.

Dinwiddie, S.H., Bucholz, K.K., 1993. Psychiatric diagnoses and self-reported child abusers. Child Abuse and Neglect 17, 465-476.

Dumaret, A.C., Coppel-Batsch, M., 1996. Evolution à l'âge adulte d'enfants placés et familles d'accueil. Psychiatrie de l'Enfant 39 (2), 613-671.

Dumaret, A.C., 2003a. Soins médico-sociaux et accompagnement psycho-éducatif. Revue de la littérature étrangère. Archives de Pédiatrie 10, 448-461.

Dumaret, A.C., 2003b. Soins précoces et guidance parentale: le point de vue des familles. Dialogue 162, 9-24.

Dumaret, A.C., 2004a. Evaluation de prises en charge précoces et d'interventions médico-sociales auprès de familles «à risque»: analyse d'une démarche exploratoire. In : Haddad, A. (Ed.), Santé Mentale du jeune enfant : prévenir et intervenir. Erès, Ramonville Saint Agne, pp. 121-131.

Dumaret, A.C., 2004b. Accompagnement des intervenants médico-psycho-sociaux et collaboration des parents et des familles d'accueil dans l'éducation des enfants. Pratiques Psychologiques 10 (1), 51-63.

Dumaret, A.C., Picchi, V., 2004c. Soins précoces : Devenir psychosocial des familles et développement des enfants. Annales Médico-Psychologiques (sous presse).

Dumaret, A.C., Titran, M. (collab. Constantin-Kuntz, M., Cousin, M., Ruffin, D.), 2004d Des stratégies de rencontre vers l'autonomie: impact à long terme d'un accompagnement médico-socio-éducatif précoce. Rapport de recherche, Villejuif.

Egeland, B., Susman-Stillman, A., 1996. Dissociation as mediator of child abuse across generations. Child Abuse Neglect 20, 1123-1132. 
Ernst, C.C., Grant, T.M., Streissguth, A.P., 1999. Intervention with high-risk alcohol and drug-abusing mothers: II three years findings from the Seattle model of paraprofessional advocacy. Journal of Community Psychology 27, 19-38.

Erickson, M.F., Korfmacher, J., Egeland, B., 1992. Attachments past and present: Implications for therapeutic intervention with mother-infant dyads. Development and Psychopathology 4, 495-507.

Ethier L.S., Biron C., Boutet M., Rivest C., 1999. Compétences parentales chez les personnes présentant des incapacités intellectuelles. Etat de la question. Revue Francophone de la déficience intellectuelle 10, 2, 109-124.

Fraser, J.A., Amstrong, K.L., Morris, J.P., Dadds, M.R., 2000. Home visiting intervention for vulnerable families with newborns: follow-up results of a randomized controlled trial. Child Abuse Neglect 24, 1399-429.

Goodman, S.H., Johnson, M.S., 1986. Life problems, social supports, and psychological functioning of emotionally disturbed and well low-income women. Journal of Community Psychology 14, 150-158.

Guédeney, N., Jacquemain, F., Glangeaud, M.C., 2000. Le rôle des facteurs environnementaux, de la vulnérabilité individuelle et du support social dans la survenue des dépressions du post-partum. Devenir 12 (4), 13-33.

Houzel, D. (dir.), 1999. Les enjeux de la parentalité. Erès, Ramonville Saint Agne.

Kumpfer, K.L., Bays, J., 1995. Child abuse and tobacco, alcohol and other drug abuse: causality, coincidence or controversy? In: Jaffe, J.H. (Ed.), The Encyclopedia of Drugs and Alcohol. MacMillan, New York, pp. 217-222.

Llewellyn, G., McConnell, D., Cant, R., Westbrook, M., 1999. Support network of mothers with an intellectual disability: An exploratory study. Journal of Intellectual \& Developmental Disability 24, 7-26.

Molénat, F., Toubin, R.M., 1996. Vers des réseaux de soins précoces. In : Dugnat, M. (Ed.), Troubles relationnels père-mère/bébé : quels soins ? Erès, Ramonville SaintAgne, 73-87.

Olds, D.L., Henderson, C.R., Kitzman, H., 1995. Effects of prenatal and infancy nurse home visitation on surveillance and child maltreatment. Pediatrics 3, 365-72.

Olds, D.L., Henderson, C.R., Kitzman, H., Eckenrode, J.J., Cole, R.E., Tatelbaum, R.C., 1999. Prenatal and infancy home visitation by nurses : recent findings. The Future of Children 9 (spring), 44-65.

Oyserman, D., Mowbray, C.T., Meares, P.A., Firminger, K.B., 2000. Parenting among mothers with a serious mental illness. American Journal of Orthopsychiatry 70 (3), 296-315.

Pan Ke Shon, J.L., 2003. Isolement relationnel et mal être. Insee Première 931.

Pianta, R., Egeland, B., Erickson, M.F., 1989. The antecedents of maltreatment: Results of the Mother-Child Interaction Research Project. In : Cicchetti, D. (Ed.), Child Maltreatment: Theory and research on the causes and consequences of child abuse and neglect. MA Cambridge University Press, Cambridge, pp. 205-253.

Potekov, T., Titran, M., 1996. Vies de familles. Un autre regard sur l'exclusion. Gallimard, Paris.

Seagull, E.A., Scheurer, S.L., 1986. Neglected and abused children of mentally retarded parents. Child Abuse and Neglect 10, 493-500.

Seitz, V., Rosenbaum, L.K., Apfel, N.H., 1985. Effects of family support intervention: a 
ten-year follow-up. Child Development 56, 376-91.

Svedin, G., Wadsby, M., Sydsjö, G., 1996. Children of mothers who are at psychosocial risk. Mental health, behavior problems and incidence of child abuse at 8 years. European Child and Adolescent Psychiatry 5, 162-171.

Titran, M., 1993. Témoignage : le groupe du mardi . Archive of Public Health 51, 135139.

Titran, M., 2004. Bientraitance parentale et grande précarité. Pratiques Psychologiques $10,65-77$.

Tymchuk, A.J., 1990. Parents with mental retardation: A national strategy. Journal of Disability Policy Studies 1 (4), 43-55.

Tymchuk, A.J., Andron, L., 1990. Mothers with mental retardation who do or do not abuse or neglect their children. Child Abuse and Neglect 14, 313-323.

Wechsler, D., 2000. Manuel de l'échelle d'intelligence pour adultes, WAIS-III (3 ${ }^{\text {ème }}$ éd.). ECPA, Paris.

Whitman, B.Y., Accardo, P., 1990. The parent with mental retardation: rights, responsibilities and issues. Journal of Social Work and Human Sexuality 8, 123136.

Zaouche-Gaudron, C., Ricaud, H., Granié, A., Beaumatin, A., 1997. Implication et différenciation des rôles éducatifs parentaux: vers de nouvelles perspectives. Revue internationale d'éducation familiale 1, 27-42. 
Tableau 1 :

\section{Situation des familles lors de l'admission au groupe et à l'enquête}

\begin{tabular}{|c|c|c|c|}
\hline \multirow[t]{2}{*}{ Caractéristiques familiales } & \multicolumn{3}{|c|}{ Familles } \\
\hline & Autonomes (12) & Stabilisées (6) & Inchangées (4) \\
\hline \multicolumn{4}{|l|}{ Antécédents familiaux } \\
\hline exposition anténatale / alcool (mères) & 6 & 5 & 3 \\
\hline $\begin{array}{l}\text { maltraitements dans l'enfance } \\
\text { (mères) }\end{array}$ & 9 & 5 & 4 \\
\hline problèmes de santé mentale (mères) ${ }^{1}$ & 5 & 5 & 1 \\
\hline addiction à l'alcool: mères & 7 & 1 & 3 \\
\hline \multicolumn{4}{|l|}{ concubins } \\
\hline violences intra-familiales $^{2}$ & 8 & 6 & 3 \\
\hline abus sexuels dans les familles ${ }^{3}$ & 7 & 4 & 3 \\
\hline membres de la famille en prison & 2 & 5 & 3 \\
\hline \multicolumn{4}{|l|}{ Participation au groupe du mardi } \\
\hline durée moyenne avec enfants $(\mathrm{m} \pm \sigma)$ & $28 \pm 12$ mois & $41 \pm 16$ mois & $22 \pm$ mois \\
\hline arrêt des $\mathrm{AEMO}^{4}$ en fin de groupe & $6 / 7$ & $2 / 6$ & $0 / 3$ \\
\hline \multicolumn{4}{|l|}{ Situation actuelle } \\
\hline ICV des mères (QIV estimé <705) & 4 & 4 & 3 \\
\hline mères abstinentes & 6 & 1 & 2 \\
\hline séparation avec le père alcoolique ${ }^{6}$ & 7 & 4 & 0 \\
\hline arrêt des violences intra-familiales & 6 & 6 & 1 \\
\hline ressources principales & salaires > RMI & $\mathrm{AAH}>\mathrm{RMI}$ & $\mathrm{AAH}=\mathrm{RMI}$ \\
\hline enfants mineurs & 33 & 17 & 12 \\
\hline familles avec mineurs placés à 1'ASE & 0 & 4 & 3 \\
\hline nouvelles AEMO à domicile & 3 & 0 & 1 \\
\hline
\end{tabular}

${ }_{3}^{1}$ la dépression, touchant la plupart des mères $\mathrm{A}$ et $\mathrm{S}$ uniquement n'est pas incluse ; ${ }^{2}$ : couple et/ou enfants ;

${ }^{3}$ événements ayant eu lieu avant, pendant et après la venue au CAMSP; ${ }^{4}$ AEMO : aide éducative en milieu ouvert ; ${ }^{5}$ une mère non coopérative n'a pu être testée, groupe $(\mathrm{Inc}) ;{ }^{6}$ un père décédé de cirrhose, un seul père est devenu abstinent et est revenu vivre en famille 


\section{Tableau 2 :}

Corrélations entre les profils des familles $\&$ la vie socio-relationnelle

\begin{tabular}{l|lll}
\hline Les 3 profils Aut, Stab, Inc & R de Spearman & $\mathrm{p}-$ level & Ordre \\
\hline \& parents (non décédés) & 0.07 & $\mathrm{nS}$ & $\mathrm{A}>\mathrm{S}>\mathrm{I}$ \\
\& famille élargie & 0.51 & 0.02 & $\mathrm{~A}>\mathrm{S}>\mathrm{I}=0$ \\
\& amis & 0.61 & 0.003 & $\mathrm{~A}>\mathrm{S}>\mathrm{I}$ \\
\& loisirs (oui/non) & 0.58 & 0.005 & $\mathrm{~A}>\mathrm{S}>\mathrm{I}$ \\
\& professionnels présents & 0.66 & 0.001 & $\mathrm{I}>\mathrm{S}>\mathrm{A}$ \\
\& capacité/demande d'aide & 0.49 & 0.02 & $\mathrm{~A}>\mathrm{S}>\mathrm{I}$ \\
\hline
\end{tabular}

Tableau 3 :

Notes standards obtenues par les 21 mères aux subtests de la WAIS

\begin{tabular}{ll}
\hline Subtests & moyennes \\
\hline Similitudes & $5,0 \pm 2,1$ \\
Vocabulaire & $4,5 \pm 2,4$ \\
Information & $3,5 \pm 1,5$ \\
Compréhension & $4,2 \pm 2,0$ \\
\hline Indice ICV & $68,0 \pm 11,3$ \\
\hline
\end{tabular}

